\title{
Tradução e adaptação de questionários norte-americanos para a avaliação de habilidades e conhecimentos na prática psiquiátrica brasileira
}

\author{
Ibiracy de Barros Camargo* \\ José Onildo B. Contel ${ }^{* *}$
}

\section{INTRODUÇÃO}

Até o final da $2^{a}$ Guerra Mundial, nos países ibero-americanos, o ensino de psiquiatria era desenvolvido em grandes hospitais psiquiátricos públicos ou privados, não seguia programas estruturados, era centrado na relação pessoal entre mestre e

Este trabalho constitui parte da dissertação de mestrado do primeiro autor, intitulada Habilidades e conhecimentos sobre a prática profissional em psiquiatria: tradução, adaptação e aplicação de questionários em psiquiatras brasileiros, apresentada à Faculdade de Medicina de Ribeirão Preto da Universidade de São Paulo em outubro de 2001, Ribeirão Preto, SP.

Trabalho apresentado na IX Jornada de Psiquiatria da Região Sul e na VI Jornada Gaúcha de Psiquiatria, julho de 2002, Porto Alegre, RS, e no XXI Congresso Brasileiro de Psiquiatria, outubro de 2003, Goiânia, GO

Departamento de Neurologia, Psiquiatria e Psicologia Médica da FMRP. USP e Programa de Pós-Graduação em Saúde Mental da FMRP-USP.

* Coordenador da Residência Médica de Psiquiatria do Hospital das Clínicas (HC-FMRP-USP) da Faculdade de Medicina de Ribeirão Preto da Universidade de São Paulo (FMRP-USP). Aluno de Doutorado do Programa de Pós-Graduação em Saúde Mental da FMRP-USP. Professor Adjunto de Psiquiatria da Universidade de Ribeirão - UNAERP. Professor Adjunto de Psiquiatria do Centro Universitário Barão de Mauá.

** Professor Associado de Psiquiatria no Departamento de Neurologia, Psiquiatria e Psicologia Médica da FMRP-USP. Professor do Programa de Pós-Graduação em Saúde Mental da FMRP-USP. Coordenador do Hospital Dia Psiquiátrico do HC-FMRP-USP. discípulo e dava ênfase a programas de países desenvolvidos. No Brasil não foi diferente: os programas na Residência de Psiquiatria começaram a tomar corpo no final da década de 1950 e começos de $1960^{1}$. Até então, muitos dos futuros especialistas procuravam por formação em países desenvolvidos, em especial Estados Unidos, Inglaterra, França e Alemanha. Essa situação mudou consideravelmente à medida que os programas nacionais sofreram modificações, de maneira a atrair e formar os futuros especialistas ${ }^{2}$.

Hoje, o número de programas brasileiros em psiquiatria credenciados na Comissão Nacional de Residência Médica (CNRM) chega a 56 e oferece 203 vagas para o primeiro ano, com programa obrigatório de 2 anos, sendo que 18 dos programas contemplam um terceiro ano optativo em subespecialidades ${ }^{3}$.

Esse crescimento na busca de especialização em psiquiatria por um número cada vez maior de médicos acompanha o que vem ocorrendo nas demais especialidades, exigindo o estabelecimento de protocolos de intenções para promover eqüidade, 
regulamentação e normatização entre as especialidades.

Os conteúdos programáticos nos 2 anos obrigatórios obedecem a normas mínimas da CNRM, com exigência de vistoria periódica. Os programas têm realizado grandes esforços para estabelecer de maneira explícita ou implícita os objetivos educacionais a serem seguidos na formação dos psiquiatras, atendendo às exigências da CNRM e às necessidades docentes e assistenciais locais ${ }^{4}$.

Esse esforço organizacional das entidades médicas inclui a busca de mecanismos institucionais permanentes para manter a qualidade do treinamento e a competência sustentada dos profissionais. A Associação Brasileira de Psiquiatria (ABP), através do Programa de Educação Médica Continuada (PEC), participa desse movimento ${ }^{5}$.

A busca de homogeneidade na formação de especialistas em substituição às duas vias existentes - a especialização por residência médica, reconhecida pela CNRM e vinculada ao Ministério da Educação e Cultura (MEC), e a especialização por avaliação da Associação Médica Brasileira (AMB) - vem sendo discutida no Conselho Federal de Medicina ${ }^{6}$.

Nos últimos 20 anos, vive-se uma psiquiatria em transição, com dramáticas mudanças na base da prática e do conhecimento psiquiátrico, no aumento do número de não-psiquiatras envolvidos no tratamento de doentes mentais e na expansão e diversificação dos locais onde se pode praticar psiquiatria. Nesse cenário de mudanças aceleradas, tem sido primordial oferecer programas de treinamento que orientem quais habilidades e conhecimentos devem predominar na prática profissional 7,8 .

Apesar de todos os esforços, surpreendentemente, pouco se sabe sobre o relacionamento entre o treinamento e a prática profissional subseqüente. Assim sendo, na medida em que a psiquiatria caminha como uma especialidade que procura ocupar uma posição de primeira ordem no cenário médico brasileiro e internacional, cada vez mais são necessárias pesquisas empíricas que ofereçam uma base científica segura na busca dessas habilidades e conhecimentos ${ }^{9}$.

No Brasil, no entanto, não existe instrumento de medida com o objetivo específico de avaliar habilidades e conhecimentos necessários à prática psiquiátrica. A importância do tema tem sido atestada pela Associação Mundial de Psiquiátrica e pela Organização Mundial da
Saúde, que se uniram em projeto cooperativo visando preparar um atlas sobre pós-graduação em psiquiatria ${ }^{10}$. O volume de publicações brasileiras indexadas tem sido insuficiente e disperso ${ }^{11-15}$; além disso, trabalhos empíricos no Brasil são escassos e não contemplam a investigação sobre o tema ${ }^{16-20}$

Com a finalidade de promover o aproveitamento de instrumentos internacionais aplicáveis em nosso meio, desde a década de 1970, tanto em psiquiatria ${ }^{21,22}$ como em outras áreas da medicina ${ }^{23}$, tem-se procedido à tradução e adaptação transcultural de instrumentos já existentes, em sua maioria oriundos de países de língua inglesa.

Este caminho tem sido considerado preferível à concepção de um novo instrumento ${ }^{24}$, por ser prático, operacional e tornar possível a comparação entre países ${ }^{25}$, permitindo a constituição de documentação internacional sobre instrumentos comuns e abrindo, assim, a possibilidade de revelar problemas relativos à diversidade de cultura e de língua de um país ou de países diferentes ${ }^{26,27}$.

Nos Estados Unidos e Canadá, as competências necessárias para a educação e a prática psiquiátricas, exigidas para fins avaliatórios desde a residência psiquiátrica até a educação médica continuada, têm merecido destaque. Algumas categorias nesse processo de avaliação são consideradas essenciais, como atendimento ao paciente, conhecimento médico, habilidades interpessoais e comunicativas, aprendizagem e aperfeiçoamento baseados na prática, profissionalismo e prática baseada em sistemas ${ }^{28-30}$

A necessidade de estudos nacionais abrangentes sobre competências essenciais à prática psiquiátrica ${ }^{31}$ dirigiu os esforços empreendidos no presente estudo para a tradução e adaptação de questionários norteamericanos $^{32}$ destinados a avaliar habilidades e conhecimentos necessários à prática psiquiátrica.

A coerência e importância dos questionários aqui analisados, atestada pela publicação em periódico internacional especializado de grande impacto, segundo critérios do ISI Web of Science, definiu a escolha do instrumento. Colaborou também nessa escolha o fato de haver 27 citações do artigo que divulgou os questionários na literatura internacional até 2001, das quais três constam no American Journal of Psychiatry, uma no British Journal of Psychiatry e uma no 
Bulletin of the Menninger Clinic, entre outras. A relevância do tema em si foi atestada pela publicação, nos últimos 5 anos, de 59 artigos relacionados ao assunto na literatura internacional. Pesou, também, a constatação da inexistência de estudo semelhante no Brasil e a experiência assistencial e docente dos autores ${ }^{33}$.

O instrumento selecionado é auto-aplicável e foi desenvolvido por Lagsley \& Yager em 1989, em pesquisa empírica com 485 chefes de departamento de psiquiatria e coordenadores de programas de residência e 499 psiquiatras na prática clínica, membros da Associação Americana de Psiquiatria, visando identificar e descrever padrões da prática e dos ideais educacionais sobre habilidades e conhecimentos que um psiquiatra deveria possuir nos Estados Unidos ${ }^{32}$.

O presente estudo está inserido num contexto crescente de busca de instrumentos padronizados que proporcionem estruturas mais formais de avaliação ${ }^{21}$. Este é o caso, por exemplo, da busca de uma linguagem internacional comum em psiquiatria, por meio de um trabalho de unificação e equivalência de linguagem diagnóstica, ou globalização de terminologia técnica, proporcionada pelos dois sistemas dominantes ${ }^{34,35}$ de classificação que têm orientado ensino, pesquisa e prática clínica psiquiátrica no mundo e no Brasil. Considerase que uma linguagem comum, estabelecida por instrumentos padronizados, permitirá que clínicos e pesquisadores dos quatro cantos do mundo falem entre $\mathrm{si}^{36}$ de maneira mais objetiva.

\section{Objetivos}

Os objetivos deste trabalho foram: 1) descrever a metodologia de tradução e adaptação da versão em português de dois questionários de avaliação de habilidades e conhecimentos em psiquiatria; 2) expor os problemas associados à tradução e adaptação dos questionários para o português; 3) disponibilizar, na íntegra, em língua portuguesa, os questionários traduzidos e adaptados.

\section{MÉTODO}

A tradução para o português foi realizada seguindo metodologia apresentada na Figura 1 , com a finalidade de obter duas versões com a maior equivalência possível, uma direta da fonte e outra com adaptações para a línguaalvo, o português, para psiquiatras brasileiros.
Sujeitos. Os autores acompanharam passo a passo todo o processo de tradução e adaptação, sendo responsáveis pela primeira versão, juntamente com o primeiro tradutor profissional. Essa versão continuou recebendo ajustes, ao ser avaliada por três psiquiatras brasileiros bilíngües, 12 psiquiatras monolíngües (quatro chefes de departamentos de psiquiatria, quatro coordenadores de residências de psiquiatria credenciadas pela CNRM e quatro especialistas em psiquiatria titulados pela $A B P$ ), resultando na versão final. $A$ retrotradução ficou a cargo de um segundo tradutor profissional, e a revisão final foi feita por um dos autores do trabalho original.

Instrumento. $O$ instrumento é constituído por dois questionários. O questionário Q1, com 48 itens temáticos, avalia habilidades, e o questionário Q2, com 51 itens, avalia conhecimentos. Ambos foram submetidos às traduções e adaptações para o português. $\mathrm{Na}$ maioria, os itens são constituídos por frases curtas, simples, com menos de 16 palavras e uma palavra-chave com a idéia dominante.

Tradução e retrotradução. A autorização de tradução e adaptação do instrumento para o português foi solicitada ao autor original, que deu sua concordância. A tradução foi realizada tomando-se por base as recomendações da Organização Mundial da Saúde ${ }^{24}$ e modelos da literatura nacional e internacional compatíveis para esse procedimento $25-27$.

\section{Estágios de modificação dos questionários} (Q1 e Q2)

Primeira versão. Q1 e Q2 foram traduzidos por tradutor profissional sem experiência no campo da psiquiatria. A seguir, os 99 itens foram revisados interativamente pelos autores, e o consenso resultante dessa primeira tradução deu origem à primeira versão em português brasileiro.

Primeira avaliação por três psiquiatras bilíngües. Essa primeira versão foi reavaliada por três renomados psiquiatras brasileiros que conviveram com a psiquiatria americana por um longo tempo e tiveram experiência com a cultura do país onde os questionários foram originalmente desenvolvidos e aplicados. Esses especialistas foram considerados capazes de reconhecer os princípios de redação dos itens e aptos a identificar o conceito que sustentou cada um deles no instrumento traduzido, do ponto de vista da equivalência lingüística. Foi estabelecido um consenso entre os três revisores e os autores, a fim de dirimir pequenas 
dúvidas, que resultaram em modificações na maneira de formular algumas questões. A seguir, essa versão em português foi submetida a um comitê de avaliadores.

Segunda avaliação por comitê de avaliadores monolíngües. Durante 1 mês, 12 avaliadores especialistas em psiquiatria revisaram Q1 e Q2 em português, procurando atualizar os mínimos detalhes dessa versão preliminar na língua-alvo.

As sugestões e comentários desses avaliadores foram incorporados como adaptações aos questionários, com melhora da compreensão de alguns itens, sem, no entanto, alterar o número deles. Nesse processo, novamente através de consenso, esses especialistas e os autores dirimiram as dúvidas existentes, o que resultou na versão final em português.
Versão final-fonte e versão-alvo dos questionários. Nesse estágio, a versão final em português foi retrotraduzida (back translation) de modo independente pelo segundo tradutor profissional para a língua fonte. Essa retrotradução foi comparada com a versão original em inglês. Os autores e este segundo tradutor de língua inglesa, através de novo consenso, avaliaram as diferenças observadas, a fim de verificar se elas afetavam a equivalência lingüística. A retrotradução resultante desse consenso foi enviada para o primeiro autor do trabalho original, para que ele a confrontasse com a versão original em inglês. Com esse procedimento, procurou-se assegurar as equivalências semântica, técnica, de conteúdo, conceitual e de critério, para que a tradução em português mantivesse os mesmos objetivos e construto da versão original.

Figura 1 - Passos metodológicos para a adaptação transcultural de questionários (Q1 e Q2) para avaliar habilidades e conhecimentos em psiquiatria

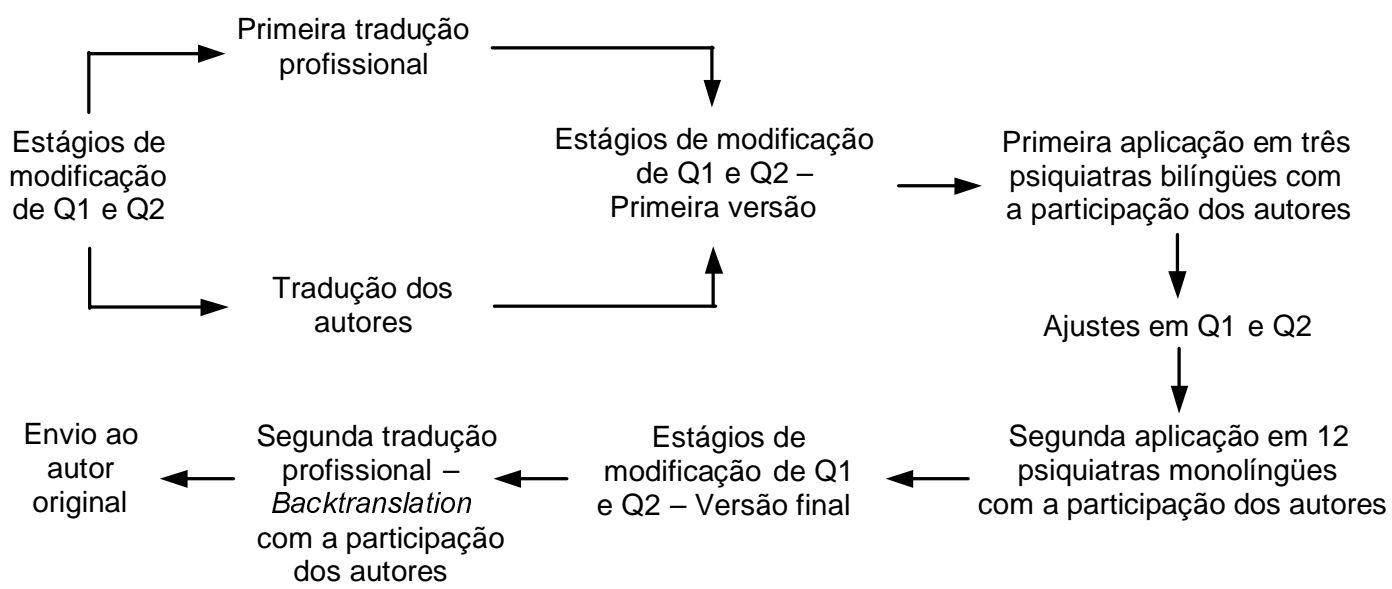

\section{RESULTADOS}

Os questionários traduzidos demonstraram possuir formato simples e fácil leitura e compreensão ao serem avaliados pelos psiquiatras bilíngües e monolíngües.

A Tabela 1 mostra 11 problemas de tradução e adaptação encontrados na busca de equivalência lingüística para a língua-alvo. Os problemas de equivalência semântica, em número de dois, correspondem às palavras "compassion" e "disorder", cujas traduções literais para o português resultaram em "compaixão" e "desordem". No lugar de "compaixão", dois avaliadores preferiram a palavra "empatia", que prevaleceu no Q1, item 5. No lugar de "desordem", preferiram a palavra "distúrbio", sendo que os autores, por consenso, acompanhando a Classificação de Transtornos Mentais e de Comportamento da CID-10, optaram pela palavra "transtorno"; tal alteração se deu no Q1, itens 24 e 30, e no Q2, itens 1, 2, 9 e 22.

As adaptações técnicas predominaram, sendo em número de cinco. Três delas 
ocorreram no Q1, como segue: no item 4, o significado da palavra "hospitalization" foi ampliado para "hospitalização integral e hospitalização parcial (hospital dia)"; no item 12, foram acrescentados "neuropsicológicos, exames de imagem de função cerebral"; e no item 16, "psicólogos e terapeutas ocupacionais". As outras duas adaptações ocorreram no Q2, como segue: no item 8 , o acréscimo de "hospitalização parcial"; e no item 11, de "aspectos da neurologia, neurobiologia, clínica médica, endocrinologia e imunologia, psiquiatricamente mais relevantes".

$\mathrm{Na}$ equivalência de conteúdo, surgiram dificuldades com a palavra "commitment", cuja tradução resultou na palavra "confinamento" a qual, na consulta final ao primeiro autor do trabalho original, resultou em "hospitalização involuntária”, expressão que foi utilizada no Q2, item 24.

Quanto à equivalência conceitual, no Q2, item 10, a palavra "bioquímicas" foi substituída por "psicobiológicas", mediante sugestão de um avaliador.

Duas modificações foram introduzidas quanto à equivalência de critério, ambas no Q2, como segue: no item 22, a expressão "distúrbios psicossomáticos" foi substituída por "transtornos dissociativos e somatoformes"; e no item 36, "ciências neurobiológicas básicas" foi substituído por "bases neurobiológicas do comportamento normal e patológico".

Tabela 1 - Síntese dos problemas de tradução e adaptação dos questionários 1 e 2

\begin{tabular}{|c|c|c|c|c|}
\hline \multirow{2}{*}{ Problemas de tradução e adaptação } & \multicolumn{3}{|c|}{ Questionários 1 e 2 adaptados } & \multirow{2}{*}{$\begin{array}{l}\text { Equivalência } \\
\text { lingüística }\end{array}$} \\
\hline & \multicolumn{2}{|c|}{ Questionários } & \multirow{2}{*}{$\frac{\text { Item(ns) }}{5}$} & \\
\hline 1. compassion/empatia & 1 & - & & Semântica \\
\hline \multirow{2}{*}{ 2. disorder/transtorno } & 1,1 & - & 24,30 & Semântica \\
\hline & - & $2,2,2,2$ & $1,2,9,22$ & \\
\hline $\begin{array}{l}\text { 3. hospitalization/hospitalização integral e hospitalização parcial } \\
\text { (hospital dia) }\end{array}$ & 1 & - & 4 & Técnica \\
\hline $\begin{array}{l}\text { 4. acréscimo: "neuropsicológicos, exames de imagem de } \\
\text { função cerebral" }\end{array}$ & 1 & - & 12 & Técnica \\
\hline 5. acréscimo: "psicólogos e terapeutas ocupacionais" & 1 & - & 16 & Técnica \\
\hline 6. acréscimo: "hospitalização parcial" & - & 2 & 8 & Técnica \\
\hline $\begin{array}{l}\text { 7. acréscimo: "aspectos da neurologia, neurobiologia, clínica } \\
\text { médica, endocrinologia e imunologia psiquiatricamente mais } \\
\text { relevantes" }\end{array}$ & - & 2 & 11 & Técnica \\
\hline 8. commitment/hospitalização involuntária & - & 2 & 24 & Conteúdo \\
\hline 9. substituição: "bioquímicas" por "psicobiológicas" & - & 2 & 10 & Conceitual \\
\hline $\begin{array}{l}\text { 10. substituição: "distúrbios psicossomáticos" por "transtornos } \\
\text { dissociativos e somatoformes" }\end{array}$ & - & 2 & 22 & Critério \\
\hline $\begin{array}{l}\text { 11. substituição: "ciências neurobiológicas básicas" por "bases } \\
\text { neurobiológicas do comportamento normal e patológico" }\end{array}$ & - & 2 & 36 & Critério \\
\hline Total de itens modificados & 6 & 10 & 16 & \\
\hline
\end{tabular}




\section{Questionário 1 - Habilidades necessárias para a prática profissional em psiquiatria}

1. Conduzir uma entrevista abrangente

2. Reconhecer problemas de contratransferência e idiossincrasias pessoais na medida em que eles influenciem a interação com pacientes e ser capaz de lidar com eles construtivamente

3. Fazer diagnósticos psiquiátricos acurados

4. Avaliar a necessidade de hospitalização integral e de hospitalização parcial (hospital dia)

5. Demonstrar interesse consistente, sensibilidade e empatia para com o paciente e sua família

6. Avaliar riscos relacionados a cada patologia, com ênfase em suicídio, homicídio e comportamento potencialmente perigoso

7. Demonstrar as qualidades de confiança, cuidado e integridade

8. Prover psicoterapia de apoio com atenção aos aspectos dinâmicos

9. Permanecer objetivo, mantendo uma postura profissional, não se envolvendo demais nem permanecendo muito distante

10. Manter registros adequados, incluindo história, estado mental, exame físico, testes diagnósticos e notas indicando o progresso do tratamento

11. Conduzir intervenção nas crises

12. Usar exames complementares apropriados, tanto testes laboratoriais como psicológicos, neuropsicológicos, exame de imagem de função cerebral e outros procedimentos diagnósticos

13. Usar adequadamente agentes psicotrópicos

14. Construir uma formulação abrangente dos problemas de pacientes físico ou psicossomaticamente doentes referidos para interconsulta e comunicá-la ao médico que os encaminhou, juntamente com sugestões práticas para o manejo

15. Conduzir psicoterapia breve

16. Desenvolver interconsulta com médicos, enfermeiros, assistentes sociais, psicólogos e terapeutas ocupacionais

17. Formular uma lista de problemas e planos de tratamento correspondentes e implementá-la através de sua responsabilidade ou encaminhamento apropriado

18. Trabalhar harmonicamente como membro de uma equipe de saúde mental na coleta de informações, planejamento e implantação do tratamento

19. Conduzir o tratamento psiquiátrico de um paciente com doença psicofisiológica ou física

20. Avaliar e tratar e/ou manejar os problemas psiquiátricos de idosos

21. Avaliar os problemas do desenvolvimento psicopatológico de adolescentes

22. Avaliar e tratar e/ou manejar alcoolismo

23. Conduzir uma entrevista diagnóstica com a família

24. Avaliar e tratar e/ou manejar transtornos alimentares

25. Avaliar e tratar e/ou manejar abuso de sedativos e/ou de psicoestimulantes

26. Ensinar conhecimentos e habilidades pertinentes à psiquiatria para estudantes de medicina e médicos

27. Avaliar e tratar e/ou manejar abuso de narcóticos

28. Conduzir terapia de família e/ou de casal em ambulatório

29. Fazer um exame clínico competente, incluindo exame neurológico detalhado

30. Avaliar e tratar e/ou manejar transtornos sexuais

31. Conduzir psicoterapia de grupo para pacientes internados

32. Administrar um programa de tratamento psiquiátrico (internação, ambulatório)

33. Conduzir psicoterapia de grupo para pacientes ambulatoriais

34. Conduzir psicoterapia em adolescentes

35. Conduzir terapia cognitiva

36. Demonstrar proficiência em psicoterapia orientada psicanaliticamente

37. Conduzir avaliação neurocomportamental

38. Conduzir tratamento psicoterápico para o paciente terminal

39. Conduzir uma avaliação completa de crianças e de suas famílias naqueles casos em que a criança é o paciente identificado

40. Conduzir avaliação psiquiátrica forense e dar testemunho por solicitação judicial sobre competência mental, sanidade e indenização para danos psicológicos ou mentais

41. Manejo dos problemas psiquiátricos de pacientes com epilepsia, lesão cerebral e outras anormalidades das estruturas cerebrais

42. Administrar ECT

43. Conduzir terapia de modificação do comportamento

44. Avaliar e tratar e/ou manejar retardo mental

45. Conduzir intervenções apropriadas em psiquiatria preventiva em nível comunitário

46. Conduzir ludoterapia

47. Conduzir pesquisa psiquiátrica

48. Usar entrevistas hipnóticas diagnósticas 


\section{Questionário 2 - Conhecimentos necessários para a prática profissional em psiquiatria}

1. Aspectos clínicos e etiológicos para transtornos psicóticos, transtornos de afeto, transtornos de ansiedade ("neuróticos") e transtornos de personalidade (incluindo transtorno borderline)

2. Critérios para diferenciar transtornos orgânicos de funcionais

3. Agentes psicofarmacológicos de uso comum: indicações, contra-indicações, mecanismos prováveis de ação, interação medicamentosa, dosagem e manejo de efeitos colaterais

4. Princípios da ética médica relacionados à psiquiatria

5. Avaliação e conduta em emergências psiquiátricas

6. Diagnóstico diferencial das síndromes psiquiátricas

7. Psiquiatria descritiva incluindo várias síndromes clínicas

8. Indicações e contra-indicações para hospitalização integral, hospitalização parcial (hospital-dia), outras intervenções, psicoterapia, terapia cognitivo-comportamental e ECT

9. Nosologia e classificação dos transtornos mentais, incluindo os transtornos mentais infantis

10. Teorias psicobiológicas mais importantes da psicopatologia

11. Aspectos da neurologia, neurobiologia, clínica médica, endocrinologia e imunologia psiquiatricamente mais relevantes

12. Aspectos psicológicos de estresse, enfrentamento e adaptação à morte, incluindo luto e outras crises vitais

13. Síndromes específicas de importância em interconsulta psiquiátrica

14. Indicações e contra-indicações para vários tipos de psicoterapia (individual, familiar, de casal, social)

15. Indicações e limitações da avaliação psicológica

16. Formulações etiológicas: psicogenéticas e psicodinâmicas, incluindo estressores desencadeantes, relacionamentos interpessoais, estrutura de personalidade e efeito da doença nos outros

17. Princípios da avaliação e intervenção em psiquiatria geriátrica

18. Princípios do crescimento e desenvolvimento humano - considerações sobre as teorias biopsicossociais mais importantes

19. Problemas psiquiátricos comuns na infância

20. Princípios para avaliação psiquiátrica de crianças e de suas famílias

21. Conceitos e teorias psicanalíticas básicas

22. Teorias sobre as patogêneses dos transtornos dissociativos e somatoformes: diagnóstico e conduta

23. Conceitos básicos de organização e comunicação em família

24. Psiquiatria forense, incluindo legislação, direitos do paciente, hospitalização involuntária, comunicação privilegiada, consentimento informado, capacidade testamentária e competência

25. Indicações, contra-indicações e teorias em relação ao mecanismo de ação do ECT

26. Princípios gerais das formas de psicoterapia mais comumente praticadas, destacando-se indicações, contra-indicações e dados sobre resultados

27. Psicoterapias: psicoterapia analiticamente orientada

28. Conceitos básicos de epidemiologia psiquiátrica

29. Adolescência com ênfase em aspectos do desenvolvimento da personalidade

30. Conceitos básicos de dinâmica de grupo

31. Principais teorias de dependência química

32. Psicoterapias: terapia de grupo

33. Princípios de intervenção em psiquiatria da criança e do adolescente

34. Psicoterapias: terapia familiar

35. Avaliação dos méritos e limitações da literatura científica

36. Bases neurobiológicas do comportamento normal e patológico

37. Modelos de interconsulta psiquiátrica

38. Fisiologia e transtornos do sono

39. Psicoterapias: terapia de casais

40. Conceitos básicos de psiquiatria social

41. Princípios básicos das teorias de aprendizagem

42. Psicoterapias: terapias cognitivas

43. Psicoterapias: modificação do comportamento

44. História da psiquiatria

45. Princípios de psiquiatria preventiva

46. Saúde mental na comunidade, possibilidade de intervenção através de consultoria e avaliação de programas

47. Problemas psiquiátricos de grupos étnicos específicos ou minorias

48. Retardo mental, incluindo avaliação e plano de tratamento

49. Psicoterapias: terapia infantil

50. Economia e psiquiatria: normas de financiamentos públicos e privados; formas de reembolso

51. Princípios de psiquiatria administrativa 


\section{DISCUSSÃO}

A pesquisa empírica em educação psiquiátrica é um campo novo e cada vez mais importante na formação de novos especialistas e na educação médica continuada, frente aos desafios de uma especialidade em transição. Nessa direção, o presente estudo segue uma tendência de tradução e adaptação, para o português, de instrumentos de medida de comportamentos e respostas relativos ao exercício da psiquiatria.

Em outras áreas da psiquiatria, são exemplos dessa tendência o desenvolvimento de instrumentos adaptados para avaliar a qualidade de vida de pacientes renais crônicos, de grande utilidade em interconsultas psiquiátricas, e de escalas específicas para a avaliação de mania, cujo objetivo é estudar a prevalência dessa doença em programas de medicina de família. Outro exemplo são as 36 escalas de avaliação de instrumentos sistemáticos de medidas em psiquiatria e psicofarmacologia, publicadas no Brasil em $1998^{37}$.

Esse interesse em pesquisa empírica tem recebido apoio governamental do Conselho de Aperfeiçoamento de Pessoal de Nível Superior (CAPES) através do fortalecimento dos cursos de pós-graduação dos departamentos de psiquiatria e saúde mental de algumas universidades públicas brasileiras.

Paralelamente ao apoio à pesquisa, têm surgido regulamentos ${ }^{38}$ oriundos do Ministério da Saúde que promovem a qualidade da assistência psiquiátrica, bem como aprimoramentos no ensino da especialização em psiquiatria através da CNRM. Por último, mas não menos importante, o Conselho Científico da AMB está aprimorando a regulamentação para emissão do título de especialista, enquanto começa a introduzir o processo de revalidação desse título.

O esforço dessas entidades em buscar o reconhecimento profissional das especialidades expressa uma crescente preocupação com a regulamentação das profissões de dentro para fora. Essas formas tradicionais de organização que centralizam diretrizes e propõem sistemas de avaliação com base nas opiniões de suas comissões apresentam o risco, quando colocadas a funcionar, de representar conceitos que podem interessar mais às agências proponentes do que aos especialistas avaliados. Tais ingerências podem vir a submeter a formação de psiquiatras e a prática da psiquiatria a diretrizes que nem sempre correspondem aos princípios aceitos pelos verdadeiros interessados.

Para contornar esse viés centralizador, cada vez mais é preciso definir indicadores locais e internos à área que orientem o diálogo e o debate para instruir a prática. Assim, tornase imprescindível obter a opinião do psiquiatra sobre sua prática atual, de modo a estabelecer critérios definidores de habilidades e conhecimentos necessários para o atendimento das solicitações do dia-a-dia de trabalho. Tal empenho investigativo empírico possibilitará direcionar os esforços voltados para a constituição de um currículo mínimo para a formação de profissionais, em sintonia com as constantes transformações da psiquiatria.

A psiquiatria, como ocorre na medicina em geral, tem uma dimensão predominantemente prática, que busca oferecer alívio ao paciente que necessita ajuda. Essa prática é suprida por dois campos de ciências básicas: as biológicas, com ênfase nas neurociências, e as psicossociais, com predomínio da antropologia, da sociologia, da psicologia social e da epidemiologia. Ao mesmo tempo em que enriquece a especialidade, essa multidisciplinaridade traz alguns complicadores, ao tornar difusos os papéis necessários ao exercício das demandas da profissão.

Diante desse quadro, o psiquiatra é o profissional de saúde mental que melhor pode integrar os achados biológicos e psicossociais. No entanto, ao atender pessoas com transtornos mentais, necessita aplicar condutas imediatas, que exigem um repertório de recursos e de técnicas terapêuticas cujos mecanismos não estão ainda suficientemente elucidados. O treinamento dos futuros especialistas e os programas de educação médica continuada podem diminuir o grau de incerteza na prática diária e ajudar a prevenir contra a ilusão da exatidão científica, que só reflete a ignorância dessas limitações.

Tendo em vista esse panorama de incertezas e transformações ${ }^{39}$ que constitui a área, os autores do trabalho original procuraram investigar diretores de programas de residência e psiquiatras na prática clínica nos Estados Unidos para saber suas opiniões sobre como se definiam enquanto formadores de opinião e especialistas em psiquiatria. Portanto, os itens do instrumento foram desenvolvidos para destacar a relevância das habilidades e conhecimentos considerados necessários aos profissionais no aprendizado e no exercício da clínica. Este foi também o objetivo dos autores 
da presente pesquisa, ao traduzir e adaptar aqueles instrumentos.

Os questionários aqui apresentados permitirão confrontar a opinião de psiquiatras de diferentes regiões geográficas do país, programas de residência, unidades psiquiátricas de hospitais gerais, hospitais psiquiátricos tradicionais, hospitais dia e centros e núcleos de atenção psicossocial, entre outras possibilidades. Abordagens qualitativas e estatísticas dos resultados poderão permitir generalizações úteis, como forma de contribuição para a elaboração de programas de ensino em programas de residência e em educação médica continuada.

O instrumento desenvolvido poderá, ainda, ser aprimorado de forma a incluir, em cada item, a opção por uma entre três respostas possíveis: "Esse item é definitivamente necessário, provavelmente necessário, ou provavelmente não necessário para a prática profissional?". Isso possibilitará uma hierarquização das opiniões dos respondentes a uma das três opções apresentadas. Ao final, seria possível saber que, quanto mais um determinado item for classificado pelos respondentes como predominantemente necessário, tanto mais os formadores de opinião nas residências médicas e nos cursos de educação médica continuada valorizarão, ou não, em termos pragmáticos, o ensino e a prática do mesmo.

Caracterizada a importância da aplicação do instrumento no Brasil, cabe agora destacar as potencialidades e as dificuldades do método utilizado na tradução e adaptação. Os 12 especialistas monolíngües foram indispensáveis, já que identificaram e adaptaram para a língua-alvo 11 problemas que passaram despercebidos pelos especialistas bilíngües. O acompanhamento de cada estágio pelos autores foi essencial para integrar e definir o rigor metodológico da tradução final.

Esse pequeno número de adaptações indica uma simetria surpreendente. Assim, o processo abrangente de tradução e adaptação descrito possibilitou que as palavras da línguafonte alcançassem uma equivalência lingüística correspondente na língua-alvo. Essa simetria sugere que se conseguiu uma adequada equivalência transcultural para os questionários. Evidentemente, certos problemas tiveram que ser enfrentados, como no caso das palavras "disorder", "psychosomatic" e "psychosomatically", conforme mostrado na Tabela 1. "disorder", cuja tradução literal é "distúrbio", constitui um bom exemplo de como uma diferença entre as línguas fonte e alvo pode distorcer completamente um significado do ponto de vista transcultural. A palavra "disorder" permaneceu no Brasil com sua tradução literal até 1994. A partir de então, seu uso foi substituído por "transtorno". Tal substituição se deve ao fato de haver, no imaginário popular, uma associação de "distúrbio" com desorganização social, desordem, tumulto, briga, violência cega, a ponto de aqueles que provocam esses problemas serem denominados desordeiros. A palavra também significa loucura, mas com conotação de grande desordem, confusão social e violência aleatória.

Do ponto de vista técnico, os autores preferiram a tradução literal do termo "psychosomatically" ("psicossomaticamante") no item 14 do Q1. Assim procederam porque a medicina psicossomática é conhecida hoje nos Estados Unidos como interconsulta psiquiátrica e psiquiatria de hospital geral aplicada em doentes físicos mais difíceis e complexos. Consultado a respeito, o autor do trabalho original considerou que "psychosomatic medicine" é sinônimo de "consultation-liaison", o que também é referendado na literatura especializada ${ }^{40}$. Por outro lado, no Q2, item 22, para a palavra "psychosomatic", os avaliadores preferiram "dissociativos e somatoformes", sob provável influência das Classificações Diagnósticas DSM-IV e CID-10. Os autores concordaram e mantiveram essa aparente contradição como expressão de um dilema antigo da psiquiatria, levantado pelo termo psicossomático, que persiste atual e ainda não pôde ser banido da prática psiquiátrica.

A grande simetria encontrada pode estar relacionada com o fato de os questionários definirem itens técnicos operacionais, específicos, claros e de interesse para uma população definida de psiquiatras nas duas línguas. Assim, não seria exagero afirmar a existência de uma similaridade surpreendente de equivalência da temática abordada pelos questionários nos Estados Unidos e no Brasil. Essa simetria parece mostrar que a versão que agora está sendo disponibilizada à comunidade científica de língua portuguesa brasileira é a mais próxima possível do instrumento original.

Algumas limitações que pesam sobre trabalhos de tradução e adaptação estão relacionadas com a imitação acrítica e mecânica de modelos importados, a exemplo do que foi indicado acima, na tradução da palavra "disorder". Essas limitações poderão ser 
contornadas na medida em que a tradução adaptada do instrumento passar por testes de campo, abrangendo uma amostragem significativa de psiquiatras brasileiros, no intuito de verificar como os profissionais reagem à aplicação dos questionários. Como limitações da tradução e adaptação do instrumento, devemos mencionar o uso de um número pequeno (apenas 12) de psiquiatras avaliadores monolíngües.

Assim sendo, a aparência de produto acabado do construto traduzido pode ser enganosa. O processo de tradução e adaptação de um instrumento é complexo e não termina na localização de uma equivalência lingüística. Em alguns aspectos, o processo é assemelhado à construção do próprio instrumento, necessitando inclusive que sejam refeitos os estudos de confiabilidade e validade em novos contextos, apoiados na fidedignidade, coerência interna e análise fatorial, entre outros procedimentos estatísticos.

Modificações futuras ou mesmo a construção de um novo instrumento devem ser levadas em conta diante da transitoriedade em campos importantes da prática e do conhecimento psiquiátrico. Os avaliadores, por exemplo, não incluíram itens que hoje pressionam a prática, como informática médica, HIV/AIDS, realidade política e econômica do atendimento em saúde mental e prática baseada em evidência.

Considerando a transitoriedade da especialidade ${ }^{39}$, é possível supor que a aplicação, de tempos em tempos, do instrumento em um número suficiente de respondentes oferecerá resultados que, quando comparados por métodos estatísticos apropriados, poderão revelar mudanças nas exigências do ensino e da prática que ainda não chegaram aos textos didáticos.

No entanto, a qualidade e a aplicabilidade do instrumento adaptado para avaliar habilidades e conhecimentos de psiquiatras brasileiros em vários níveis só poderão ser confirmadas em pesquisa de abrangência nacional, que será objeto de um segundo estudo.

\section{CONCLUSÕES}

O presente estudo procura diminuir as barreiras existentes para a condução de estudos experimentais na pesquisa educacional psiquiátrica, ao oferecer instrumento de âmbito internacional com essa finalidade. Diferenças na origem e estrutura das línguas portuguesa e inglesa exigiram um trabalho em profundidade para manter a qualidade do instrumento durante o processo de tradução e adaptação. O método comum de tradução e retrotradução mostrou-se insuficiente e precisou ser acompanhado de revisão de cada estágio pelos autores e de acompanhamento por psiquiatras avaliadores bilíngües e monolíngües. Avanços atuais na psiquiatria impossibilitam que se aceite qualquer paradigma isolado para explicar ou orientar a pesquisa e a prática tanto atuais como futuras. Diante disso, qualquer instrumento de investigação, na melhor das hipóteses, estará retratando o momento atual. Considerando a dinâmica de transformações que tem acompanhado a prática da psiquiatria, modificações futuras do presente instrumento poderão ser necessárias. Um estudo com tal abrangência, como ocorreu nas aplicações em grande escala nos Estados Unidos, poderá permitir uma definição empírica de quais habilidades devem ser adquiridas e quais conhecimentos são necessários, e até prioritários, para as tarefas cada vez mais diversificadas e complexas da prática psiquiátrica em nosso país.

\section{REFERÊNCIAS BIBLIOGRÁFICAS}

1. Camargo IB, Vansan GA, Contel JOB. História do início do ensino de psiquiatria na residência médica do $\mathrm{HC}$ FMRP-USP. J Bras Hist Med 2002;5(1):38.

2. Guimón J, editor. Formation dês psychiatres de demain. Paris: Masson; 1998.

3. Brasil, MEC/CNRM/SESU. Relação de instituições e programas de residência médica por região. Brasília: MEC/CNRM/SESU; 2001.

4. Brasil, MEC/CNRM/SESU. Resolução No 1. Critérios Básicos para Credenciamento de Programas de Residência Médica. Brasília: MEC/CNRM/SESU; 2002.

5. ABP. Programa de educação médica continuada. Psiquiatr Hoje 2003;2:14

6. Normativa para emissão de títulos de especialista e certificados de áreas de atuação. JAMB 2004;1331:12.

7. Rifkin B. Changes and dilemmas in psychiatric practice. In: Silberman EK, editor. Successful psychiatric practice. Washington DC: American Psychiatry Press; 1995. p.113.

8. Lieberman JA, Rush J. Redefining the role of psychiatry in medicine. Am J Psychiatry 1996;153(11):1388-97.

9. Okasha A. The future of medical education and teaching: a psychiatric perspective. Am J Psychiatry 1997;154(Suppl.):6.

10. WPA-WHO. Atlas project on postgraduate training in psychiatry. Available at: http//www.who.int/ mental_health/en/. Accessed 2003.

11. Kerr-Corrêa F. Residência em psiquiatria na Faculdade de Medicina de Botucatu: relato crítico de um programa. Rev Bras Educ Med 1986;10(3):148-51.

12. Calil LC, Contel JOB. Estudo dos programas de residência médica em psiquiatria no Estado de São Paulo em 1993. Rev Bras Psiquiatr 1999;21(3):139-44. 
13. Elkis $H$. Residência médica: competências mínimas e psiquiatria moderna. Rev Bras Psiquiatr 1999;21(3):1378.

14. Rodrigues CRC. A especialização em psiquiatria. Revista ABP-APAL 1989;11(4):155-9.

15. Camargo IB, Contel JOB. Research methodologies to asses teaching in psychiatric residency: a literature review. Rev Bras Psiquiatr 2003;25(3):160-5.

16. Eizirik CL, Fonseca AF, Gazal CH, Ferreira ED, Costa FM, Goldim JR, et al. O psiquiatra do Rio Grande do Sul e sua prática de consultório: perfil dos entrevistados (1). Rev Psiquiatr RS 1991;13(3):160-6.

17. Eizirik CL, Costa FM, Goldim JR, Santos TM, Gazal C Zampel R, et al. Perfil e prática profissional de psiquiatras no Brasil. Revista ABP-APAL 1996;18(2):49-52.

18. Eizirik CL, Goldim JR, Fonseca AF, Gazal CH, Costa FM Piltcher R, et al. Uma amostra de psiquiatras do Rio Grande do Sul e São Paulo: perfil demográfico e prática de consultório. Revista ABP-APAL 1993;15(3):82-6.

19. Bastos O, Martins MCCA. Esboço de um perfil atual do psiquiatra pernambucano. Rev ABP 1979;2(1):19-29.

20. Cabrera CC. O tratamento manicomial e a desinstitucionalização, segundo opiniões de psiquiatras de Ribeirão Preto [tese de doutoramento]. Ribeirão Preto: Faculdade de Medicina de Ribeirão Preto, USP; 2000.

21. Jorge MR. Adaptação transcultural de instrumentos de pesquisa em saúde mental. In: Gorestein C, Andrade LHSG, Zuardi AW, editores. Escalas de avaliação clínica em psiquiatria e em psicofarmacologia. São Paulo: Lemos; 2000, p.53-8.

22. Shansis F, Berlim MT, Mattevi B, Maldonado G, Izquierdo I, Fleck M. Desenvolvimento da versão em português da escala administrada pelo clínico para avaliação de mania (EACA-M): "Escala de Mania de Altman". Rev Psiquiatr RS 2003;25(3):412-24.

23. Duarte PS, Miyazaki MCOS, Ciconelli RM, Cesso R Tradução e adaptação cultural do instrumento de avaliação de qualidade de vida para pacientes renais crônicos (KDQUOL-SFTM). Rev Assoc Med Bras 2003;49(4):375-81.

24. Cardoso CS, Bandeira M, Caiaffa WT, Fonseca JOP Escala de qualidade de vida para pacientes com esquizofrenia (QLS-BR): adaptação transcultural para o Brasil. J Bras Psiquiatr 2002;51(1):31-8.

25. Vallerand $R$. Vers une methodologie de validadtion transculturelle de questionnaires psychologiques. Can Psychol 1989;30:662-80.

26. Hunt SM, Alonso J, Bucquet DN, McKenna WI. Crosscultural adaptation of health measures. Health Policy $1991 ; 19(2): 33-44$.

27. Massoubre C, Lang F. La traduction des questionnaires et des tests: techniques et problèms. Can J Psychiatry 2002;47(1):61-7

28. Persad E, Leverette J. Training issues in psychiatry in Canada. Can J Psychiatry 2003;48(4):213-4.

29. Scheiber SC, Kramer TAM, Adamowski SE. The implications of core competencies for psychiatric education and practice in the US. Can J Psychiatry 2003;48(4):215-21.

30. Miller SI, Scully JH, Winstead DK. The evolution of core competencies in psychiatry. Acad Psychiatry 2003;27(3):128-30.

31. Botega NJ. Residência de psiquiatria no hospital geral: uma enquête nacional. J Bras Psiquiatr 1991;40(8):419-22.

32. Langsley DG, Yager J. The definition of psychiatrist: eight years later. Am J Psychiatry 1988;145(4):469-75.

33. Contel JOB. A supervisão no processo de ensino de psiquiatria dinâmica e psicoterapia: retrospecto crítico de 30 anos da experiência pessoal. Medicina $1992 ; 25(3): 330-43$.
34. American Psychiatric Association. Diagnostic and statistical manual of mental disorders (DSM-4). 4th ed. Washington DC: American Psychiatric Press; 1995

35. OMS. Classificação de transtornos mentais e de comportamento da CID-10. Porto Alegre: Artes Médicas 1993.

36. Berganza CE. Broadening the international base for the development of an integrated diagnostic system in psychiatry. World Psychiatry 2003;2(1):38-40.

37. Gorestein C, Andrade LHSG, Zuardi AW. Escalas de avaliação em psiquiatria e psicofarmacologia. São Paulo: Lemos; 2000.

38. Brasil. Portaria GM/MS nํ 2048. Regulamento técnico dos sistemas estaduais de urgência e emergência. Diário Oficial da União, Brasília, 5 nov. 2002.

39. Weissman $\mathrm{SH}$, Nadelson. Psychiatry in transition. In Dikstein L, Riba M, Oldham JM, editors. Review of psychiatry. Washington DC: American Psychiatry Press; 1996. vol. 15, p.503-5

40. Gitlin DF, Levenson JL, Lyketsos CG. Psychosomatic medicine: a new psychiatric subspecialty. Acad Psychiatry 2004;28:4-11.

\section{RESUMO}

Introdução: A bibliografia brasileira não faz referência a instrumentos e guias padronizados de investigação sobre a prática e o ensino de psiquiatria. Como contribuição ao tema, os autores traduziram e adaptaram instrumento norte-americano para avaliar habilidades e conhecimentos em psiquiatria. Os objetivos deste estudo foram: 1) descrever a metodologia para a elaboração de versões em português de questionários de avaliação de habilidades e de conhecimentos em psiquiatria; 2) expor os problemas de tradução e adaptação dos questionários; 3) disponibilizar os questionários na íntegra em língua portuguesa. Método: A metodologia compreendeu os seguintes estágios: primeira versão profissional; avaliação por psiquiatras bilíngües; avaliação por psiquiatras monolíngües; versão final em português; retrotradução independente por segundo tradutor profissional; revisão final pelo primeiro autor do trabalho original. Todos os estágios foram revisados sistematicamente pelos autores. Resultados: Foram encontrados 11 problemas de tradução e de adaptação na busca da equivalência lingüística, com predomínio de cinco problemas de equivalência técnica. Discussão: A pesquisa empírica em educação psiquiátrica é um campo novo e cada vez mais importante na formação de novos especialistas e na educação médica continuada frente aos desafios de uma especialidade em transição. Conclusão: O pequeno número de adaptações pode significar que a tradução e a adaptação das palavras da língua fonte alcançaram uma equivalência lingüística. O método comum de tradução e retrotradução foi insuficiente e precisou ser acompanhado pela avaliação de psiquiatras bilíngües e monolíngües e pela revisão de cada etapa pelos autores. O presente estudo disponibiliza em língua portuguesa instrumento único para avaliação de habilidades e conhecimentos na prática psiquiátrica brasileira. 
Descritores: Tradução, retrotradução, questionários, habilidades, conhecimentos, psiquiatria, prática profissional, psiquiatria em transição.

\section{ABSTRACT}

Introduction: The Brazilian literature does not make reference to standardized instruments and guidelines aimed at the assessing psychiatric teaching and practice. As a contribution to the theme, the authors of the present study translated and adapted a North American instrument designed to evaluate skills and knowledge in the psychiatric practice. The objectives of this study were: 1) to describe the methodology used to translate two questionnaires on skills and knowledge of psychiatry; 2) to summarize the difficulties faced in the process of translating and adapting the questionnaires; 3) to make the questionnaires available in Portuguese. Method: The methodology comprised the following stages: first professional version; evaluation by bilingual psychiatrists; evaluation by monolingual psychiatrists; final version in Portuguese; independent back translation by a second professional translator; final review by the first author of the original paper. The authors systematically followed all stages. Results: Eleven of the translation and adaptation problems faced during the process referred to linguistic equivalence, whereas five problems were related to technical equivalence. Discussion: Empirical research in psychiatric education is a new and increasingly important field. It will improve the quality of education and training in both residency and continuing education programs in psychiatry - a specialty in transition. Conclusion: The small number of adaptations may indicate that the translation and adaptation of the words in the source language reached linguistic equivalence. The use of translation and back translation proved to be insufficient, and the process had to be followed by bilingual and monolingual psychiatrists and also by the authors. This paper offers the scientific community the first instrument in the Portuguese language aimed at assessing skills and knowledge in the Brazilian psychiatric practice.

Keywords: Translation, back translation, questionnaires, skills, knowledge, psychiatry, professional practice, psychiatry in transition.

Title: Translation and adaptation of North American questionnaires for the assessment of skills and knowledge in the Brazilian psychiatric practice

\section{RESUMEN}

Introducción: La bibliografía brasileña no menciona instrumentos y guías estándar de investigación sobre la práctica y la enseñaza de psiquiatría. Para complementar algún conocimiento al tema, fueran traducidos y adaptados instrumentos estadounidenses para evaluar las habilidades e conocimientos de psiquiatría. Objetivos: 1) describir la metodología para la elaboración de una versión en portugués de cuestionarios de evaluación de habilidades y conocimientos en psiquiatría; 2) exponer los problemas de traducción y adaptación de los cuestionarios; 3) disponer los cuestionarios, de forma íntegra en lengua portuguesa. Método: La metodología comprende de las siguientes etapas: Primera versión profesional; evaluación por psiquiatras bilingües y por psiquiatras monolingües; versión final en portugués; una nueva traducción independiente por otro traductor profesional y una revisión final por quien hizo el trabajo original. Todas las etapas fueran sistemáticamente revisadas por los autores. Resultados: Se encontraron once problemas de traducción y de adaptación en la búsqueda de equivalencias lingüísticas, predominando cinco problemas de equivalencia técnica. Discusión: La pesquisa empírica en educación psiquiátrica es un campo nuevo y cada vez se hace más importante en la formación de nuevos especialistas y en la educación médica continua, confrontando así los desafíos de una especialidad en transición. Conclusión: El pequeño número de adaptaciones puede significar que la traducción y la adaptación de las palabras de la lengua fuente alcanzaron una equivalencia lingüística. El método común de traducción y retraducción fue insuficiente y necesitó ser acompañado por evaluaciones de psiquiatras bilingües y monolingües junto a una revisión de cada etapa por los autores. El presente estudio pone a disposición en lengua portuguesa un instrumento único para la evaluación de las habilidades y conocimientos en la práctica psiquiátrica brasileña.

Palabras clave: Traducción, retraducción, cuestionarios, habilidades, conocimientos, psiquiatría, práctica profesional, psiquiatría en transición.

Título: Traducción y adaptación de cuestionarios estadounidenses para evaluar habilidades y conocimientos en la práctica psiquiátrica

Correspondência:

Dr. Ibiracy de Barros Camargo

Rua Rui Barbosa, 1661

CEP 1415-120 - Ribeirão Preto - SP

Fone: (16) 636.3660

Fax 166259209

E-mail ibiracy@uol.com.br

Copyright (@) Revista de Psiquiatria do Rio Grande do Sul - SPRS 